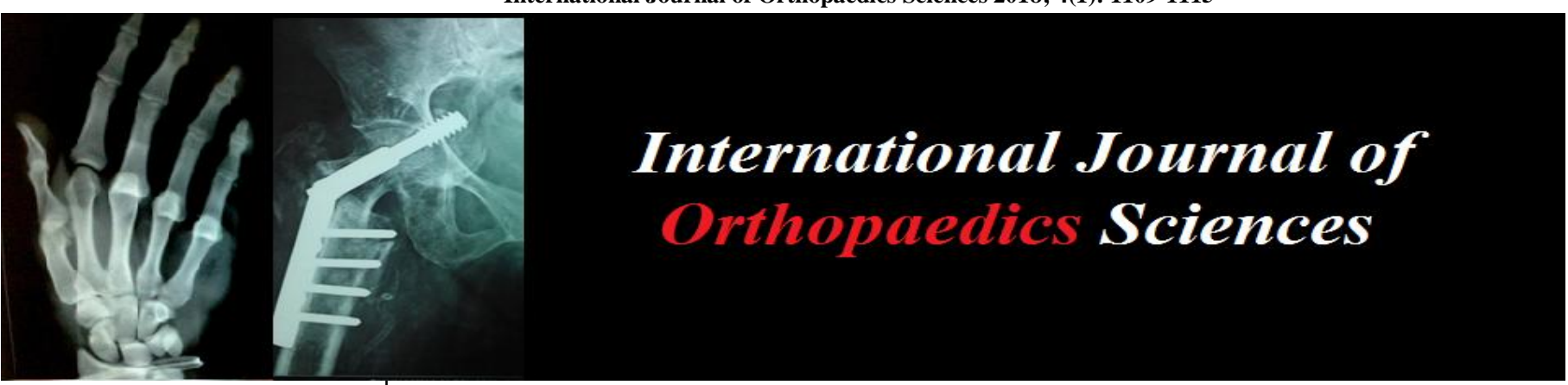

ISSN: $2395-1958$

IJOS 2018; 4(1): 1109-1115

(C) 2018 IJOS

www.orthopaper.com

Received: 16-11-2017

Accepted: 18-12-2017

Dr.Rahul Jaju,

Assistant Professor, Dept. of Orthopaedics, Govt. Medical

College Aurangabad,

Maharashtra, India

Dr. Ansari Muqtadeer Abdul Aziz

Head of Unit \& Associate

Professor, Dept. of Orthopaedics,

Govt. Medical College

Aurangabad, Maharashtra, India

Dr.Vaibhav Sadigale

Consultant Orthopaedic surgeon,

Pandharpur, Maharashtra, India

Correspondence

Dr.Ansari Muqtadeer Abdul Aziz

Head of Unit \& Associate

Professor, Dept of Orthopaedics, Govt. Medical College,

Aurangabad, Maharashtra, India

\section{Functional results of arthroscopic bankart's Repair (ABR) in bankart's lesion of the shoulder}

\author{
Rahul Jaju, Ansari Muqtadeer Abdul Aziz and Vaibhav Sadigale
}

DOI: https://doi.org/10.22271/ortho.2018.v4.i1p.156

\section{Abstract}

Background: Shoulder pain due to shoulder instability is a disabilitating cause in patients aged 15 to 40 years who are active. Shoulder instability in the anterior direction is most commonly due to post traumatic shoulder dislocation. The basic cause is a Bankart lesion which has historic significance. Treament has evolved from open surgery which is vast, extensive to minimally invasive arthroscopic surgery nowadays most commonly preferred by patients all over as well as surgeons, with good to excellent results.

Materials and Methods: A total of 48 patients were included in the study. The age of the patients varied from 17 to 44 years with a mean of 27.31 years. There were 45 males and 3 females in our study. This study was Prospective and Retrospective study Conducted at our working place between July 2008 to April 2010. The University of California and Los Angeles (UCLA) shoulder rating scale is used to assess the functional outcomes of the patients operated with ABR.

Results: In our series of 48 patients were having preoperative instability ,out of which $37 \%$ patients were improved in terms of no postoperative instability. Two patients with fair UCLA scores at 6 months or later had poor compliance with physiotherapy protocol. There was no case of anchor pull out around the shoulder or same shoulder revision surgery for anterior glenohumeral instability.

Conclusion: Arthroscopic Bankart repair (ABR)is a safe, effective, cosmetic treatment method with good clinical outcomes, excellent postoperative shoulder motion and low recurrence rates.

Keywords: ABR-Arthroscopic Bankart's repair, UCLA-University of California and Los Angeles, VASVisual Analog Scale, shoulder instability

\section{Introduction}

Shoulder instability can be particularly troubling to patients by causing them pain, weakness and shoulder dysfunction. It is one of the most unstable joints of the human skeletal system ${ }^{[1]}$. In 1920 Bankart published a paper stating that acute dislocations cause the humeral head to force anteriorly out of the glenoid cavity and tear the fibro cartilaginous labrum from almost the entire anterior half of the rim of glenoid cavity, as also the capsule and periosteum from anterior surface of the neck of scapula. This traumatic detachment of glenoid labrum has been called the Bankart's lesion. ${ }^{[2]}$ Patients with recurrent shoulder dislocation undergo destructive changes of glenoid labrum and humerus. Bankart's lesion repair is one of the most frequent procedure performed in shoulder surgery. Earlier treatment of Bankart's lesion in recurrent shoulder dislocation was open repair with surgery like open Bankart's or Bristow's or PuttiPlat operation. The treatment of recurrent shoulder dislocation has travelled a long way, starting with complete open repair, to arthroscopic assisted mini -open techniques to complete arthroscopic repair ${ }^{[3]}$ The treatment of recurrent shoulder dislocation has travelled a long way, starting with total open repair, to arthroscopic assisted mini-open techniques to complete arthroscopic repair. The surgical fundamentals emphasised substantially improved the reliability of the outcomes of repairing Bankart's lesion by arthroscopy ${ }^{[4-6]}$ These include preservation or meticulous repair of glenoid labrum, adequate decompression of the subacromial space by resection of any antero-inferior osteophytes, surgical release as necessary to obtain freely mobile muscle-tendon units, secure fixation of the tendon to the greater tuberosity, and closely supervised rehabilitation including early passive motion within a protected range. 
The treatment of Bankart's dislocation lesion in recurrent shoulder has changed dramatically during the recent past as there is progression towards less invasive arthroscopic means to obtain equivalent or better results to the traditional open procedures. As of today, Arthroscopic Bankart's repair is technically demanding as most of the patient's techniques and suture materials being designed and techniques such as double row anchors to overcome past inadequacies. Arthroscopic Bankart's repair has several advantages ${ }^{[6]}$ With this technique it is possible to use a smallest incision and to protect the deltoid muscle. It provides diagnosis and treat the intraarticular lesions; soft tissue damage is minimized, thus, postoperative pain decreases, rehabilitation is facilitated and the risk of adhesive capsulitis is decreased. This study has been undertaken to assess the short term functional outcome of Arthroscopic Bankart's repair in patients with anterior recurrent shoulder dislocation using UCLA score.

\section{Materials and Methods}

This study was Prospective and Retrospective study Conducted at Sancheti Institute of Orthopaedics and Rehabilitation between July 2008 to April 2010.

Criteria for Inclusion:

All patients between 15 to 40 years of age with:

1. Recurrent anterior shoulder dislocation

2. Lesion confirmed by MRI.

\section{Exclusion Criteria}

\section{Patients with}

1. Rotator cuff tear,

2. Revision Bankart's,

3. past history of fracture upper end humerus,

4. Instability with absent labrum or Bony procedure,

5. Multi Directional Instability

6. Habitual Dislocation patients.

\section{Treatment Protocol}

During the period between January 2007 and November 2009, all patients treated with arthroscopic Bankart's repair for anterior glenohumeral instability at the Sancheti Institute were screened using the inclusion and exclusion criteria. Informed consent was taken for all patients that fit the inclusion criteria and all patients willing to undergo the assessment were included.

\section{Pre-Operative Planning}

1. X rays of the shoulder -True AP and Stryker Notch view

2. MRI Scan of the involved shoulder.

3. Pre-operative routine laboratory investigations.

All cases in this series were performed by a single surgeon using a standard technique using titanium suture anchors.

\section{Surgical Technique}

Surgery was performed in lateral position and under general anaesthesia. The arm was on traction with light weight of about $3 \mathrm{~kg}$ on a draped support. Three portals were used. Posterior portals were used as viewing portal for standard 4 $\mathrm{mm}$ arthroscope (the viewing portals), while anteromedial and anterolateral porals were used for the instruments. The patient was placed in the lateral decubitus position on a beanbag support to tilt the trunk approximately $20^{\circ}$ posteriorly. We created posterior, anterosuperior, and anterior midglenoid portals. The posterior portal was made approximately $2 \mathrm{~cm}$ inferior and $1 \mathrm{~cm}$ medial to the acromial angle. The anterosuperior portal was made just inferior to the anterior edge of the acromion with the guidance of a transarticular
Wissinger rod. The anterior midglenoid portal was placed just superior to the leading edge of the subscapularis tendon. A small cannula was inserted into the anterosuperior portal, and a large-diameter threaded cannula (8.4- $\mathrm{mm})$ was placed in the anterior midglenoid portal. Diagnostic arthroscopy was performed through the posterior and anterosuperior viewing portals. Looking through the anterosuperior portal, the surgeon mobilized the anterior capsulolabraltissue from the anterior glenoid surface using a Liberator knife. Mobilization was carried out beyond the 6 o'clock position. The subscapular is muscle was exposed in order to achieve good mobility of the capsulolabral tissue. The soft tissue on the medial wall of the glenoid was removed with use of a radiofrequency device. Light decortications by abrading the medial wall of the glenoid was done with a 4-mm convex rasp (Linvatec), which was inserted through the anterior midglenoid portal. With 2-mm pituitary forceps, small pilot markings were created on the margin of the anterior glenoid rim. A bone punch was inserted through the anterior midglenoid portal with the tip of the punch pointed at the lateral apex of the pilot marking; this created the anchor hole a few millimeters lateral to the corner of the glenoid. We created a funnel-shaped screw hole using a special bone punch that had side blades at the base of its tip. The funnelshaped screw hole was directed as vertically as possible, so that it would not penetrate the glenoid cortical bone, while creating the most inferior screw hole at the 5:30 position for a right shoulder. This was done by keeping the tip of the bone punch in the pilot marking and pivoting the shaft of the bone punch inferiorly and laterally Vertical orientation of the screw hole can reduce the possibility of screw penetration of the inferior glenoid cortex, which often occurs during drilling with a power drill. Also the funnel-shaped hole provided a wide space for the suture material to slide well while the sliding-type of knots were being tied because of less friction between the suture and the margin of the aperture of the screw hole17. A mini-Revo screw, $2.7 \mathrm{~mm}$ in diameter and $8.5 \mathrm{~mm}$ in length, or a FASTak screw, $2.4 \mathrm{~mm}$ in diameter and 11.7 $\mathrm{mm}$ in length, loaded with a number- 2 nonabsorbable polyester suture was manually inserted into the hole. At the final turn of the screw handle, the orientation of the eyelet of the screw was aligned parallel to the future suture direction in order to further reduce the friction between the suture material and the screw. With use of a suture hook loaded with a Shuttle Relay a capsular suture was created about $1 \mathrm{~cm}$ inferior Arthroscopic appearance of the right shoulder as seen through the anterosuperior portal shows mobilization of the anterior capsulolabral tissue from the anterior glenoid wall. The subscapularis muscle should be exposed to achieve adequate mobilization of the anterior capsulolabral tissue. The Liberator knife was inserted through the midglenoid port to the anchor and at or below the level of the glenoid surface. The suture hook, with the capsular tissue, was shifted proximally to the point of the suture anchor, and then the suture hook was passed under the labrum. This eliminated any redundant pouch anterior to the glenoid wall. Although we routinely performed a capsular shift, the degree of capsular shift was individualized according to the intraoperative measurement of capsular laxity. Patients with less anterior translation (grade 1+ or lower), a type-1 anterior labrum, or a distinct anterior capsular ligament underwent a less extensive capsular shift. The Shuttle Relay was retrieved through the anterosuperior portal. One end of the suture was engaged in the eyelet of the Shuttle Relay and then was pulled back out of the midglenoid portal. Finally, a sliding knot was made. 
The knots were created on the capsular side. The second and third suture anchors were done at the 4.00 and 3.00 o'clock positions for the left shoulder and 8.00 and 9.00 o'clock position for the right, in the same manner. The sutures were tied using the Tennessee slider knot, which is easy to tie, has a low profile, and possesses good holding strength secured by a series of three reversing half-hitches on alternating posts ${ }^{[10]}$.

\section{Post -Op Protocol}

All patients were given shoulder arm pouch. Immediate post op. I.V antibiotics would be given for 2 days i.e on the day of the surgery and $1^{\text {st }}$ post op day. They were discharged on the next day after dressing.

\section{Rehabilitation}

Physiotherapy was started on post op day 1 or 2.It was started with scapular retraction exercises and rhomboids. Active assisted exercises were allowed till 90 degree. Abduction and extension exercises were also started as much as possible for the patients. Strictly active cuff exercises were not done till 6 weeks. Elbow and wrist exercises were also started immediately. All these exercises were followed for 6 weeks after which PHASE ONE of the shoulder rehabilitation was started. It included exercises in the form of full can exercises for 5 days. Empty can exercises were started after 5 days. On day 1 levator exercises were started, followed by serratus anterior exercises without theraband on day 2, and with theraband on day 3. Scapular exercises of the rhomboids, serratus anterior and retractors were followed. Cuff strengthening was also done. After 10 days of start of phase 1 , ROM exercises in the form of rotation of shoulder were done.

\section{Criteria for Functional Assessment}

The University of California and Los Angeles (UCLA) shoulder rating scale is used to assess the functional outcomes of the patients operated with ABR. This scale is considered more detailed and yet simple and easier to use in terms of assessment of range of motion and strength, taking only a few minutes to complete. Out of a possible 35 points, the score includes up to 10 points each for pain and function, and up to 5 points each for range of active forward elevation, strength of forward elevation and patient satisfaction. The UCLA score has a high $(15 \%)$ component of patient satisfaction that is all or none. So if a patient is not satisfied with the results they drop the scores by $15 \%{ }^{[7-9]}$

\section{Statistical Analysis}

For continuous variables like range of motion - forward flexion and abduction, mean and standard deviation is calculated in each of the categories of injury-surgery interval, age, fracture morphology and gender. Mean between categories is then compared by t-test and $p$ value is calculated. For scores like UCLA, non-parametric Mann-Whitney test is used to compare the distribution between categories. To compare the percentage distribution of UCLA scores between categories, Chi square test is used and $p$ value is calculated. In all three cases, a $p$ value less than $0.05(p<0.05)$ is considered statistically significant.

\section{Results}

A total of 48 patients were included in the study. The age of the patients varied from 17 to 44 years with a mean of $27.31 \pm$ 6.1 (17-44) years. There were 45 males and 3 females in our study. The Injury-surgery interval the time since first dislocation was $2.38 \pm 1.29$ years range being 1 to 7 years. The age at first dislocation was $25.40 \pm 5.34$ with range of
(16-43). All 48 patients were followed with mean time 13.71 months $(2-26)$ months. We have also calculated extent of lesion in terms of minutes of tear with mean $19.62 \pm 8.4$ (337). UCLA score at final follow up was $28.29 \pm 1.20(17-$ 35). There are 14 patients which were involved in sports activities. We have classified activities in terms of grade of activities:

Grade 1: Very severe limitation of activities $(<70 \%$ of preinjury level),

Grade 2: Is severe limitation of activities $(70 \%-79 \%$ of preinjury level),

Grade 3: Is moderate limitation of activity level (80\% - 89\% of preinjury level),

Grade 4: Is mild limitation of activities (90\% - 99\% of preinjury level),

Grade 5: Is no limitation of activities with $100 \%$ of preinjury level.

In our study we had one patient with grade 3 activity, 38 patients with grade 4 activity and 8 patients with grade 5 activity. (Seung Ho Kim et al 2005) (Table 1)

Table 1

\begin{tabular}{|c|c|}
\hline Variables & Mean \\
\hline Age & 27.31 years $\pm 6.1(17-44)$ \\
\hline Male : Female & $45: 3$ \\
\hline Dexterity; Right:left & $34: 14$ \\
\hline Sports involvement & 14 \\
\hline No of dislocation & $3.96 \pm 1.96(1-10)$ \\
\hline Injury - Surgery Interval & 2.38 years $\pm 1.29(1-7$ years $)$ \\
\hline Age at first dislocation & $25.40 \pm 5.34(16-43)$ \\
\hline Preop rom & $98.13 \pm 10.243(70-110)$ \\
\hline No. of suture anchors. & $2.46 \pm 1.01(1-6)$ \\
\hline Follow up time in months & 13.71 months $(2-26$ months $)$ \\
\hline UCLA scores at final follow up & $28.29 \pm 1.20(17-35)$ \\
\hline Forward Flexion at final follow up & 118.33 degrees \\
\hline Minutes of tear & $145-180$ degrees $)$ \\
\hline Infection & $19.62 \pm 8.4(3-37)$ \\
\hline Dislocation & 0 \\
\hline
\end{tabular}

Average UCLA score at final follow-up for the patients was 28.29 with 6 patients completing 2 years follow up, 10 patients completing 18 months follow up, 32 patients completing 1 year follow up. At the final follow up, there was significant improvement in UCLA with significant $\mathrm{P}$ value of 0.005.46 shoulders were rated with good to excellent results with a UCLA score of $>27$. Amongst the remaining 2 shoulders, 1 had a final follow up at 4 months or less and further follow up of these patients could not be done during the study duration. The UCLA score has a high (15\%) component of patient satisfaction - that is all or none. So if a patient is not satisfied with the results they drop the scores by $15 \%$. The Visual Analog Scale (VAS) scores improved from a mean of $6.58( \pm 0.709)$ pre-operatively to a mean of 2.37 ( \pm 1.4) post-operatively. This is a $74.37 \%$ change or improvement which is statistically significant, $\mathrm{p}$ value $<0.05$. The average forward flexion range of motion at final follow up was $118.33 \pm 40.5$ degrees (Range 45 - 180 degrees) which is not significanty different from the pre-operative mean (table). There is a wide range in the minimum \& maximum ranges achieved. In our series of 48 patients $62.5 \%$ patients were having preoperative instability out of which $37 \%$ patients were improved in terms of no postoperative instability (Table 2). 2 patients with fair UCLA scores at 6 months or later had poor compliance with physiotherapy 
protocol. There was no case of anchor pull out around the shoulder or same shoulder revision surgery for anterior glenohumeral instability. The age, injury to surgery interval and gender of the patient did not seem to have a significant effect on the UCLA at the final follow up

Table 2

\begin{tabular}{|c|c|c|c|}
\hline & Mean ${ }^{* *}$ & SD & P value \\
\hline UCLA & & 1.90 & 0.005 \\
\hline preop & $29.93 \pm 1.90$ & \multirow{2}{*}{2.10} & \\
\hline postop & $31.14 \pm 2.10$ & & \\
\hline \multicolumn{4}{|l|}{ VAS score } \\
\hline preop & $6.58 \pm 0.87$ & 0.87 & \multirow{2}{*}{0.000} \\
\hline postop & $2.37 \pm 1.4$ & 1.40 & \\
\hline \multicolumn{4}{|c|}{ Power/grade } \\
\hline preop & $98.15 \pm 5.3$ & 5.30 & \multirow{2}{*}{0.193} \\
\hline postop & $118.33 \pm 4.8$ & 4.8 & \\
\hline \multicolumn{4}{|l|}{ Instability } \\
\hline preop & $62.5 \%$ & & \\
\hline postop & $25 \%$ & & \\
\hline
\end{tabular}

Final instability

Total 48 patients were operated of which 36 patients were having no instability whereas 12 patients were having instability at final follow-up.

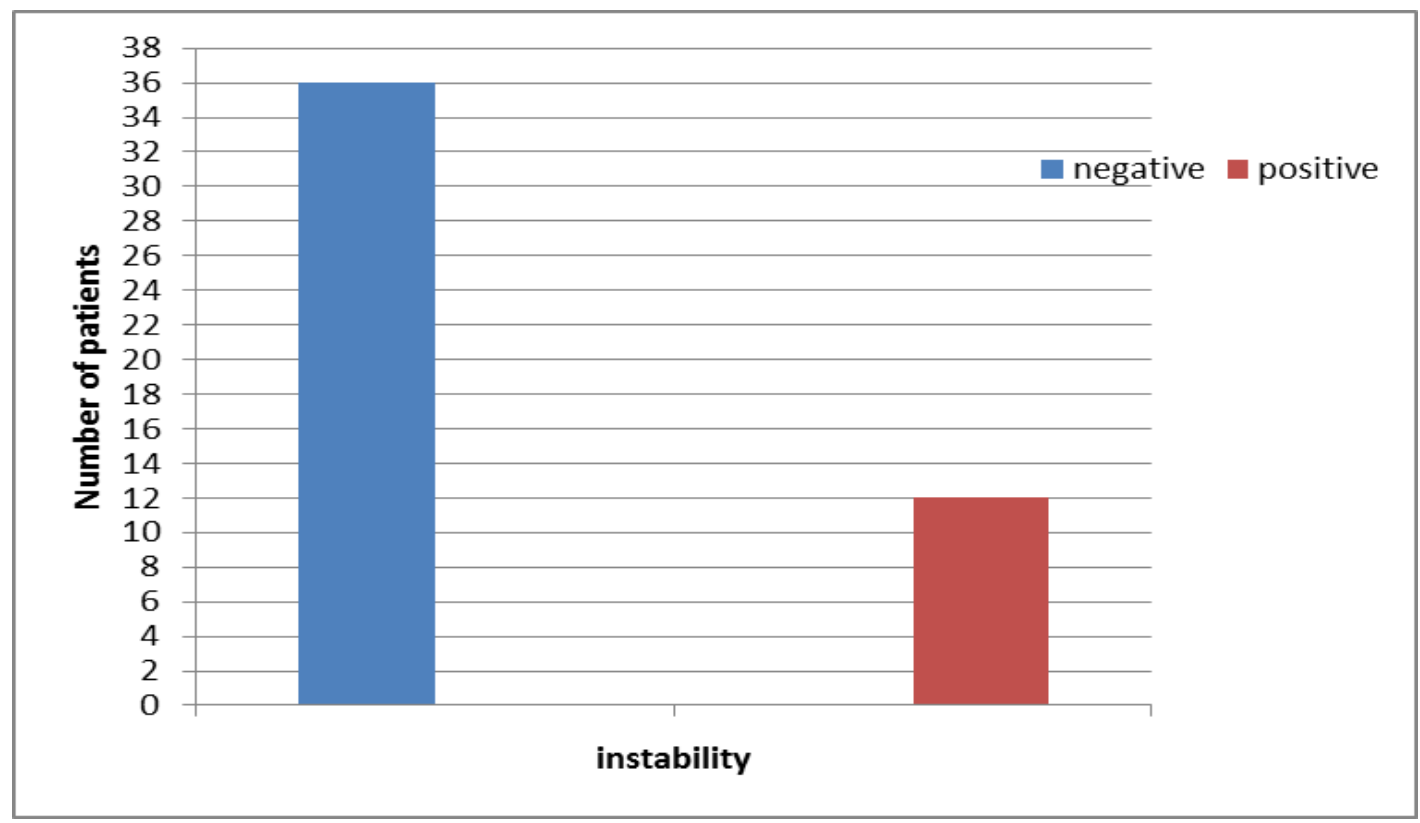

Fig 1

\section{Minutes of tear}

In our study we calculated extent of lesion in glenoid in terms of minutes of tear. Of the 48 patients, 30 had a tear minutes Less than 20 and 18 had a more than 20 minutes. The minutes of tear, the extent of tear, has significant correlation with total number of dislocation with $31 \%$ difference between two different groups, number of suture anchors and relative difference of forward flexion (Table 8). The minutes of tear, the extent of tear, has significant correlation, number of suture anchors with $\mathrm{p}$ value $<0.005$ and relative difference of forward flexion. Whereas tear minutes does not have statistically significant correlation with duration of instability, age at first dislocation, UCLA, VAS score and activity status.

Table 8

\begin{tabular}{|c|c|c|c|c|}
\hline Total dislocations & & \multicolumn{2}{|c|}{ Tear minutes } & Total \\
\hline & & $<=20$ & $>20$ & \\
\hline \multirow[t]{2}{*}{$<=3$} & Number & 20 & 6 & 26 \\
\hline & $\%$ within Total dislocations & $76.9 \%$ & $23.1 \%$ & $100.0 \%$ \\
\hline \multirow[t]{2}{*}{$>3$} & Number & 10 & 12 & 22 \\
\hline & $\%$ within Total dislocations & $45.5 \%$ & $54.5 \%$ & $100.0 \%$ \\
\hline \multirow[t]{2}{*}{ Total } & Number & 30 & 18 & 48 \\
\hline & $\%$ within Total dislocations & $62.5 \%$ & $37.5 \%$ & $100.0 \%$ \\
\hline
\end{tabular}




\section{Discussion}

Of all the different types of uni and multi directional instability of the shoulder joint, the most common type is anterior glenohumeral instability ${ }^{[11,12]}$. As the mnemonic goes TUBS-traumatic unidirectional Bankart's Repair, And AMBRII - atraumatic multidirectional. It usually affects young adults secondary to traumatic shoulder dislocation. Rowe and Zarins reported a rate of $95.6 \%$ traumatic origin to anterior dislocation in their study that included 500 patients. ${ }^{13}$ Peeling off the anterior labrocapsuloligamentous structure from the anterior glenoid removes the deepening, socket stabilising, effect of the glenoid labrum ${ }^{[14]}$. The standard tretament involves restoring the place and integrity of this labrocapsuloligamentous structure to the articular surface,thereby deepening the glenoid cavity and stabilising the humeral head by giving more volume. This is achieved by using sutures and suture anchors, which can be done either open or arthroscopically ${ }^{[15,16]}$. Capsular laxity is the another reason for glenohumeral instabilityfailure to diagnose capsular laxity preoperatively can lead to failure of repair [17, ${ }^{18]}$ For a perfect shoulder instability repair result, all the facts causing instability must be understood and treated appropriately. Previous arthroscopic techniques involved using transglenoid sutures or bio-absorbable tacks ${ }^{[19]}$. literature has shown open cases to cause postoperatice subscapularis defiency ${ }^{[20,21]}$. Arthroscopic Bankart repair is a minimally invasive cosmetic approach with less surgical trauma and less blood loss, improvements in operating time, less perioperative morbidity, minimum hospital stay, time loss from work, and decrease number of complications together with a lower cost of surgerywith accelerated post-operative rehabilitation. ${ }^{[22-24]}$ All the patients have good post-operative range of movement with excellent external roration making them capable to do all jobs and sports ${ }^{[25-27]}$

UCLA system of scoring is widely used with much historic significance hence we have used that score in our studies as well as its simplicity of application on OPD basis by the examining clinician makes its wider use for evaluation acceptable ${ }^{[28-30]}$ Hiroyuki sujaya also observed good results in patients aged less than 50 years. ${ }^{31}$ In our study, we have observed significantly better functional outcomes in terms of UCLA scores in all patients except two patients which are having fair results. $(p<0.05)$. However, there was statistically significant difference in the range of motion in terms of forward flexion and abduction at final follow up. We did not find any statistically significant difference in groups operated within 1 year or later than 1 year. The gender of the patient also, did not seem to have a bearing on the functional results following surgery or the final range of motion achieved. Our analysis shows that age at first dislocation had no correlation with total number of dislocation, minutes of tear, post-op VAS and UCLA score, ROM and final instability. Preoperatively, all patients i.e 14 out of 48 patients who had been participating in sports activity had been returned to there preinjury level at least $90 \%$ of activity. Boileau, had similar experiences in the past ${ }^{[32]}$ Out of 29 patients having preoperative subjective instability has 25 patients were not having subjective instability post op. There was significant improvement in UCLA score and VAS score postoperatively. All cases included in the study were primary cases and 46 (85.7\%) of the 48 cases completing a one year follow up had good or excellent results. Of the remaining 2 cases, the functional outcomes were fair, on the subsequent follow up.
This is an important feature that we would like to reinforce. Arthroscopic bankarts repair; we believe is a soft tissue surgery. It is the surgeons' ability to restore the original biomechanics \& render same tension amongst the glenohumeral muscles \& ligaments. Once the soft tissues have scarred down after previous surgery or even immobilisation, it is beyond the best surgeon to restore normal biomechanics. Along with soft tissue tension, the importance of the suture anchors is paramount. The minutes of tear has significant correlation with total number of dislocation, number of suture anchors and relative difference of ROM. However it does not have statistically significant correlation with duration of instability, age at first dislocation, UCLA, VAS score and activity status.Poor results were reported in many studies in the past in terms of recuuence of instability. Subsequent studies using have reported better outcomes in the view of ROM, instability and activity status. Duration of instability does not have significant correlation with post-operative instability and relative difference of VAS, UCLA score and total number of dislocation. We found no correlation with number of dislocation with relative difference of activity forward flexion, VAS and UCLA. Hiroyuki sujaya also observed good results in series of 109 patients. In this study, supervised active stretching and strengthening exercises of the shoulder were begun after two to three weeks of immobilization. Thus, it is possible that our systematic and supervised shoulder rehabilitation programme complements the standard surgical technique to give such good to excellent functional outcomes. In this study, we have compared the intraoperative factors which influence the eventual outcome of the surgery such as Hill Sach's lesion, extent of tear which is calculated in terms of minutes of tear. In our study, we have not had a single case of anchor pull out and Dislocation. We did not have a single case of infection in our series. We are unsure that these excellent short term results achieved in our study are enduring and will last for a patient's lifetime. This would need a much longer follow up to comment on the recurrence of instability. Also, the number of cases included in this series is small. It would require larger number of cases to report if similar results are reproducible in a larger group of patients.

\section{Conclusion}

Arthroscopic Bankart's Repair (ABR) for anterior glenohumeral instability with Bankart's lesion gives good early functional results. The results seem to be better in the form of better ROM, recurrence rate and activity status. However, with good surgical technique, using suture anchors and appropriate post-operative rehabilitation the morphology of tear, injury - surgery interval and sports activity of the patient do not have a bearing on the eventual functional outcomes or the range of motion and stability achieved.

Limitations: A longer follow up is required to comment if these excellent short term outcomes are enduring and to assess the subsequent instability and recurrence of dislocation. Also, a larger case series is needed to report if similar results are reproducible in majority of the patients.

Conflict of interest : nil

Funding: nil

Case: 35 years Male. Operated with right arthroscopic bankarts repair 


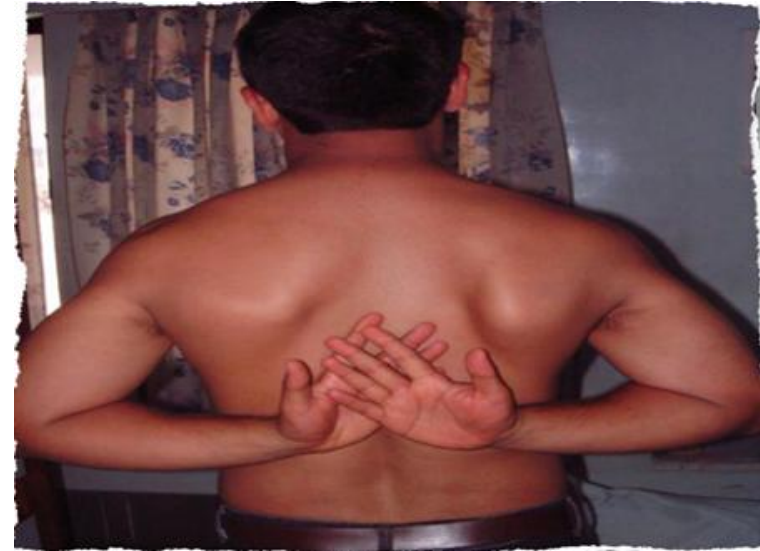

A

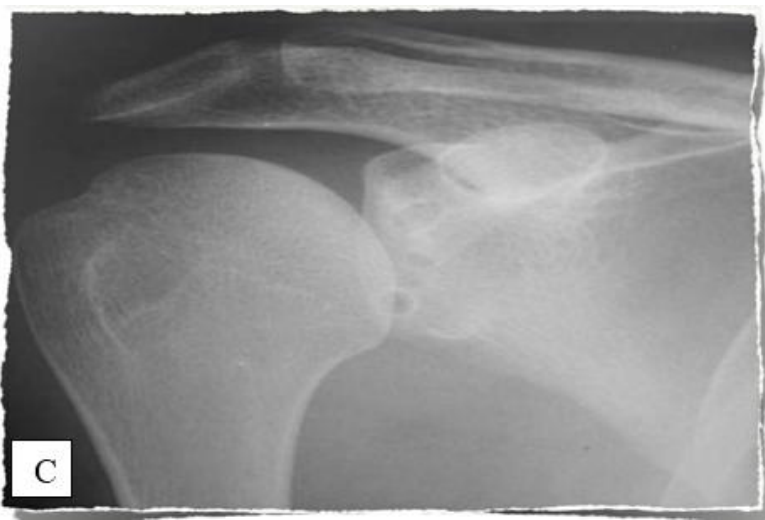

Preop

\section{Reference}

1. Erkoçak ÖF, Yel M. The functional results of arthroscopic bankart repair with knotless anchors for anterior glenohumeral instability. Eur J Gen Med. 2010; 7:179-86.

2. Bankart AS. Recurrent or habitual dislocation of the shoulder. BMJ 1920; 1:1132-3.

3. Sedeek SM, Tey IK, Tan AH. Arthroscopic bankart repair for traumatic anterior shoulder instability with the use of suture anchors. Singapore Med J. 2008; 49:676-81

4. Sperling JW, Smith AM, Cofield RH, Barnes S. Patient perceptions of open and arthroscopic shoulder surgery. Arthroscopy. 2007; 23:361-6.

5. Fabbriciani C, Milano G, Demontis A, Fadda S, Ziranu F, Mulas PD. Arthroscopic versus open treatment of Bankart lesion of the shoulder: A prospective randomized study. Arthroscopy. 2004; 20:456-62.

6. Ee GW, Mohamed S, Tan AH. Long term results of arthroscopic bankart repair for traumatic anterior shoulder instability. J Orthop Surg Res. 2011; 6:28.

7. Amstutz HC, Sew Hoy AL, Clarke IC. UCLA anatomic total shoulder arthroplasty. Clin Orthop Relat Res 1981; 155:7-20.

8. Ellman H, Hanker G, Bayer M. Repair of rotator cuff. End-result study of factors influencing reconstruction. J Bone Joint Surg Am. 1986; 68:1113-44.

9. Ellman H. Arthroscopic subacromial decompression: Analysis of one- to three-year results. Arthroscopy. 1987; 3:173-81.

10. Amit Mishra, Pulak Sharma, Deepak Chaudhary. Analysis of the functional results of arthroscopic Bankart repair in posttraumatic recurrent anterior dislocations of shoulder,Indian Journal of Orthopaedics Year : 2012; 46(6):668-674

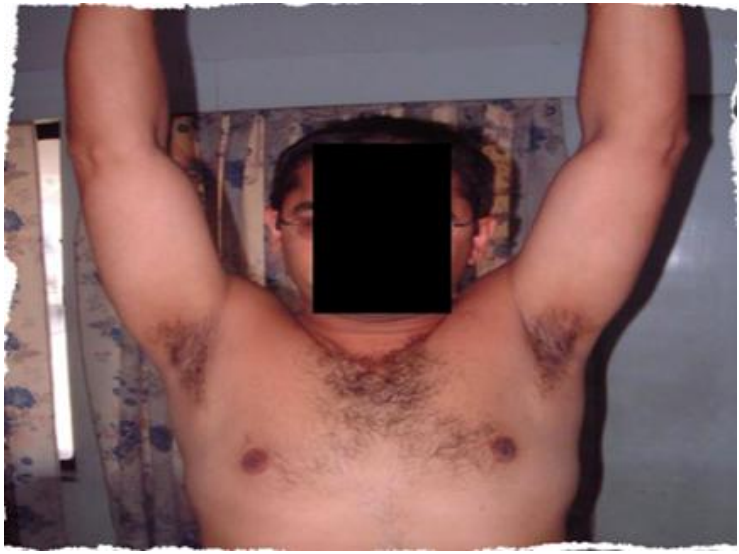

B

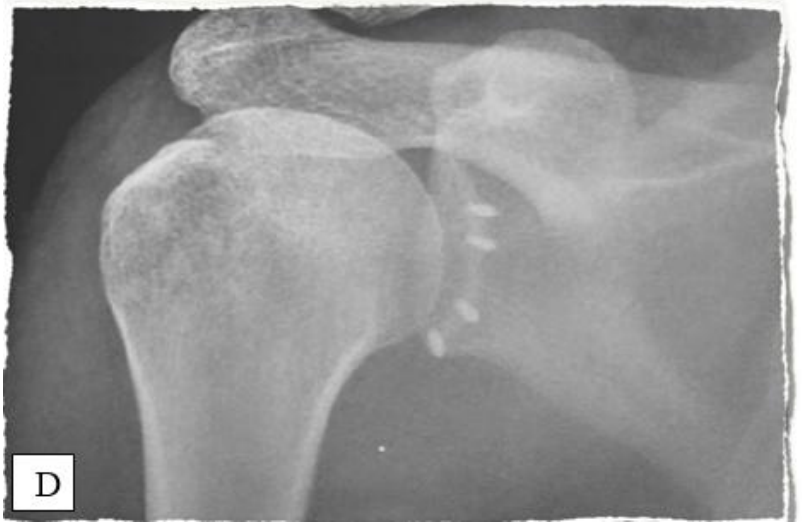

Postop

11. Baker CL, Uribe JN, Whitman C. Arthroscopic evaluation of acute initial anterior shoulder dislocation. Am J Sports Med. 1990; 18:25-8.

12. Liu SH, Henry MH. Anterior shoulder instability. Current review. Clin Orthop. 1996; 323:327-37.

13. Rowe CR, Zarins B. Recurrent transient subluxation of the shoulder. J Bone Joint Surg. 1981; 63A:863-72.

14. Cooper DE, Arnoczky SP, O'Brien SJ, Warren RF, DiCarlo E, Allen AA. Anatomy histology and vascularity of the glenoid labrum. J Bone Joint Surgery Am. 1992; $74: 46-51$.

15. Lippitt SB, Vanderhooft JE, Harris SL, Sidles JA, Harryman DT, Matsen FA. Glenohumeral stability from concavity compression: A quantitative analysis. J Shoulder Elbow Surg. 1993; 2:27-35.

16. Kirkley A, Griffin S, Richards C, Miniaci A, Mohtadi N. Prospective randomized clinical trail comparing the effectiveness of immediate arthroscopic stabilization versus immobilization and rehabilitation in first traumatic anterior dislocation of the shoulder. Arthroscopy. 1999; 15:507-14.

17. Levine WN, Arroyo JS, Pollock RG, Flatow EL, Bigliani LU. Open revision stabilization surgery for recurrent anterior glenohumeral instability. Am J Sports Med. 2000; 28:156-60.

18. Zabinski SJ, Callaway GH, Cohen S, Warren RF. Revision shoulder stabilization: 2 to 10 year results. J Shoulder Elbow Surg. 1999; 8:58-65.

19. Freedman KB, Smith AP, Romeo AA, Cole BJ, Bach BR Jr. Open bankart repair versus arthroscopic repair with transglenoid sutures or bioabsorbable tacks for recurrent anterior instability of the shoulder. Am J Sports Med 2004; 32:1520-7.

20. Scheibel M, Tsynman A, Magosch P, Schroeder RJ, 
Habermeyer P. Postoperative subscapularis muscle insufficiency after primary and revision open shoulder stabilization. Am J Sports Med. 2006; 34:1586-93.

21. Bottoni CR, Smith EL, Berkowitz MJ, Towle RB, Moore JH. Arthroscopic versus open shoulder stabilization for recurrent anterior instability. Am J Sports Med. 2006; 34:1730-7.

22. Wang C, Ghalambor N, Zarins B, Warner JJ. Arthroscopic versus open bankart repair: Analysis of patient subjective outcome and cost arthroscopy. Arthroscopy. 2005; 21:1219-22.

23. O'Neill BD. Arthroscopic Bankart repair of anterior detachments of the glenoid labrum. A prospective study. Arthroscopy. 2002; 18:755-63.

24. Kim SH, Ha KI, Cho YB, Ryu BD, Oh I. Arthroscopic anterior stabilization of the shoulder: Two to six year followup. J Bone Joint Surg Am. 2003; 85:1511-8.

25. McIntyre LF, Caspari RB, Savoie FH. The arthroscopic treatment of multidirectional shoulder instability: Twoyear results of a multiple suture anchor. Arthroscopy. 1997; 13:418-25.

26. Savoie FH, Miller CD, Field LD. Arthroscopic reconstruction of traumatic anterior instability of the shoulder. The Caspari technique. Arthroscopy 1997; 13:201-9.

27. Gartsman GM, Roddey TS, Hammerman SM. Arthroscopic treatment of anterior-inferior glenohumeral instability. Two to five-year followup. J Bone Joint Surg Am 2000; 82:991-1003.

28. Plancher KD, Lipnick SL. Analysis of evidence-based medicine for shoulder instability, arthroscopy. Arthroscopy. 2009; 25:897-908.

29. Wright RW, Baumgarten KM. Shoulder outcomes measures. J Am Acad Orthop Surg 2010; 18:436-44.

30. Godfrey J, Hamman R, Lowenstein S, Briggs K, Kocher M. Reliability, validity, and responsiveness of the simple shoulder test: Psychometric properties by age and injury type. J Shoulder Singapore Med J. 2008; 49:681.

31. Hiroyuki Sugaya. Techniques to evaluate glenoid bone loss,Curr Rev Musculoskelet Med. 2014; 7(1):1-5

32. Boileau P, Villalba M, Héry JY, Balg F, Ahrens P, Neyton L. Risk factors for recurrence of shoulder instability after arthroscopic Bankart repair. J Bone Joint Surg Am. 2006; 88(8):1755-63. 